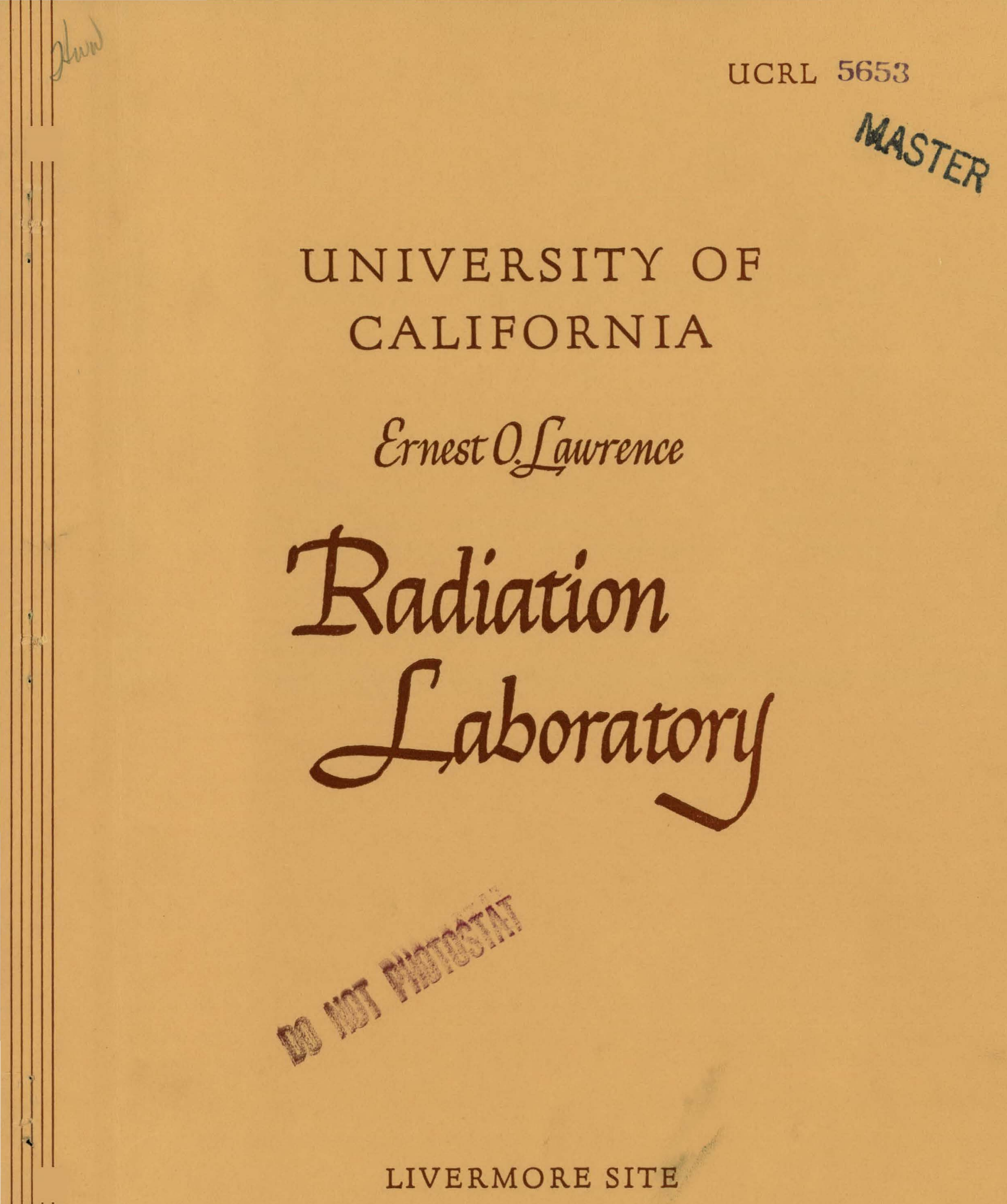




\section{DISCLAIMER}

This report was prepared as an account of work sponsored by an agency of the United States Government. Neither the United States Government nor any agency Thereof, nor any of their employees, makes any warranty, express or implied, or assumes any legal liability or responsibility for the accuracy, completeness, or usefulness of any information, apparatus, product, or process disclosed, or represents that its use would not infringe privately owned rights. Reference herein to any specific commercial product, process, or service by trade name, trademark, manufacturer, or otherwise does not necessarily constitute or imply its endorsement, recommendation, or favoring by the United States Government or any agency thereof. The views and opinions of authors expressed herein do not necessarily state or reflect those of the United States Government or any agency thereof. 


\section{DISCLAIMER}

Portions of this document may be illegible in electronic image products. Images are produced from the best available original document. 
1

\section{UNIVERSITY OF CALIFORNIA \\ Lawrence Radiation Laboratory \\ Livermore, California}

Contract No. W-7405-eng-48

\section{APPLICATION OF A MODIFIED DEBYE-HŮCKEL} THEORY TO FULLY IONIZED GASES

R. E. Kidder and H. E. DeWitt

August 14, 1959

Printed for the U. S. Atomic Energy Commission 


\section{APPLICATION OF A MODIFIED DEBYE-HÜCKEL}

THEORY TO FULLY IONIZED GASES *

R. E. Kidder and H. E. DeWitt

Lawrence Radiation Laboratory, University of California

Livermore, California

\section{ABSTRACT}

The equations of the Debye-Hückel theory, modified to include quantum statistics, are discussed. It is found that the nonlinear equations used by Cowan and Kirkwood are not unique, and that the nonlinear theory can be formulated in different ways to give different answers. The linearized equations of these alternative formulations are discussed, and the correct form of the linearized theory is established. From the linear theory, the Helmholtz free energy of a slightly degenerate plasma is derived, and from this result, useful formulae in the near-classical limit are obtained for the pressure and internal energy.

* This work was performed under the auspices of the U. S. Atomic Energy Commission. 


\title{
APPLICATION OF A MODIFIED DEBYE-HÜCKEL THEORY TO FULLY IONIZED GASES
}

\author{
R. E. Kidder and H. E. DeWitt \\ Lawrence Radiation Laboratory, University of California \\ Livermore, California
}

\section{INTRODUCTION}

In its linearized form, the classical Debye-Hückel theory of ionic solutions has, been very useful in providing a quick derivation of the thermodynamic properties of a fully ionized gas in the classical limit $(\hbar=0)$. The results of this intuitive theory have been confirmed by a rigorous statistical-mechanical derivation using the Mayer cluster expansion of the canonical partition function. It has been shown that the linearized form of the Debye theory is equivalent to the summation of the ring integrals of the classical cluster expansion. The theory in its nonlinear form, however, is beset with insurmountable difficulties when applied to a classical multicomponent gas of point-charged particles. From the success of the Thomas-Fermi statistical theory of the atom, it has been expected that by modifying the' Debye-Hückel theory to the extent of . describing the clcctrons witl Feirii slatistics instead of Boltzmann statistics the difficulties of the classical theory could be removed. Recently, Kirkwood and Cowan have made detailed numerical calculations of the thermodynamic functions of a dense plasma, using the nonlinear equations of a Debye-HückelThomas-Fermi theory. ${ }^{2}$ Also, Landau and Lifshitz have indicated how the linearized Debye theory may be amended to include the effects of quantum

\footnotetext{
${ }^{1}$ E. W. Montroll and J. E. Mayer, J. Chem. Phys. 9, 626 (1941).

${ }^{2}$ R. D. Cowan and J. G. Kirkwood, J. Chem. Phys. 29, 264 (1958).
} 
statistics. ${ }^{3}$ Exact results for the thermodynamic functions of a slightly degenerate gas are now available. ${ }^{4}$ In this note we examine the Kirkwood-Cowan theory in its linearized form. It is found that the logic used by them enables one to write two alternative sets of equations which give different answers for the same problem. Landau and Lifshitz use a slightly different set of equations and obtain results that disagree with the exact theory because of the violation of a symmetry requirement. We take the point of view that the Debye-Hückel theory, when modified with quantum statistics, should, in its linearized form, agree with the rigorous results obtained by summing the ring diagrams of the quantum-mechanical grand partition function. A form of the linearized theory that meets this requirement is given.

\section{THE BASIC APPROXIMATIONS AND THE EQUATIONS OF THE DEBYE-H ÜCKEL-THOMAS-FERMI THEORY}

We consider a fully ionized gas composed of electrons with average density $n_{-0}$ and ions of charge $z_{i} e$ and average density $n_{+0}$. Electrical neutrality requires that $z_{i} n_{\text {to }}$ be equal to $n_{-0}$. Around each ion there is an average potential $\Psi_{+}$due to the ion being a center surrounded by a statistical distribution of positive and negative charge; similarly, a round each electron an average potential $\Psi_{-}$is defined; These average potentials satisfy Poisson's equation:

$$
\begin{aligned}
& \nabla^{2} \Psi_{+}=-4 \pi z_{i} \text { e } \delta(r)-4 \pi \rho_{+}, \\
& \nabla^{2} \Psi_{-}=4 \pi \text { e } \delta(r)-4 \pi \rho_{-} .
\end{aligned}
$$

${ }^{3}$ L. D. Landau and E. M. Lifshitz, Statistical Physics Pergaman Press, 1958), pp $229-33$.

${ }^{4}$ H. E. DeWitt, Thermodynamic Functions of a Partially Degenerate, Fully Ionized Gas, University of California Radiation Laboratory Report No. UCRL5652, Aug. 1959. 
The $\delta$ functions express the boundary conditions, which are:

$$
\begin{aligned}
& \lim _{r \rightarrow 0} r \Psi_{+}=z_{i} e \\
& \lim _{r \rightarrow 0} r \Psi_{-}=-e .
\end{aligned}
$$

The average charge densities are given by:

$$
\begin{aligned}
& \rho_{+}=e\left(z_{i} n_{++}(r)-n_{-+}(r)\right), \\
& \rho_{-}=e\left(z_{i} n_{+-}(r)-n_{--}(r)\right),
\end{aligned}
$$

where $n_{++}(r)$ and $n_{-+}(r)$ are the average particle-number densities of ions and electrons, respectively, at a distance $r$ from a given ion. Similarly, $\mathrm{n}_{+-}$and $\mathrm{n}_{-}$are the densities of ions and electrons about a given electron. The average distribution of negative charge about an ion must be the same as the average distribution of positive charge about an electron; hence, we have the basic symmetry requirement:

$$
n_{-+}(r)=z_{i} n_{+-}(r)
$$

Since the electrons obey Fermi statistics, the average density of electrons about ions may be written as:

$$
n_{-+}(r)=\left\langle\sum_{p_{i}}\left[1+\exp \left(-a_{e}+\beta p_{i}^{2} / 2 m+\beta w_{-+}(r, n)\right]^{-1}\right\rangle\right.
$$

where $w_{-+}(r, a)$ denotes the energy of an electron at a distance $r$ from an ion, in the presence of all the remaining ions and electrons in a particular configuration denoted by $a$. The averaging in (5) is taken over all configurations, with the distance $r$ held fixed. An initial basic assumption of the Debye-Huckel approach is that Eq. (5) is approximated by:

$$
n_{-+}(r) \cong \sum_{p_{i}}\left[1+\exp \left(-a_{e}+\beta p_{i}^{2} / 2 m+\beta\left(w_{-+}(r, a)\right\rangle_{a}\right)\right]^{-1} \text {. }
$$


This assumption is rigorously true only to first order in $w_{-+}(r, a)$, since there is no reason to expect that $\left\langle\begin{array}{c}n \\ w_{+}\end{array}\right\rangle_{a}$ is equal to $\left\langle w_{-+}\right\rangle_{a}^{n}$ for $n$ greater than 1. Henceforth, the average electron-ion energy, $\left\langle w_{-+}(r, a)\right\rangle_{a}$, will be denoted by $w_{-+}(r)$. If the summation of $(6)$ over momentum states $p_{i}$ is converted to an integration, the following equation, in the notation of the previous paper is obtained:

$$
n_{-+}(r)=n_{-0} b_{e}^{-1} \cdot l_{1 / 2}\left(a_{e}-\beta w_{-+}(r)\right)
$$

where

$$
\begin{aligned}
\zeta_{e} & =\frac{(2 \pi \hbar)^{3} n-0}{\left(2 s_{e}+1\right) \pi^{3 / 2}(2 m k T)^{3 / 2}} \\
d_{1 / 2}(a) & =\frac{4}{\sqrt{\pi}} \int_{0}^{\infty} \frac{d x x^{2}}{1+\exp \left(-a+x^{2}\right)}
\end{aligned}
$$

Similarly, for the ions, we find:

$$
n_{++}(r)=n_{+o} \zeta_{i}^{-1} \begin{cases}\ell_{1 / 2}\left(a_{i}-\beta w_{++}(r)\right) & \text { F.D. } \\ g_{1 / 2}\left(a_{i}-\beta w_{++}(r)\right) & \text { B.E. }\end{cases}
$$

depending on whether the ions are fermions or bosons. The charge density must vanish as $r$ approaches $\infty$, since the system is electrically neutral; this requirement. determinee the cliemical potentials $a_{e}$ and $a_{i}$ in (7) and (9) from the equations:

$$
\begin{aligned}
& \zeta_{e}=d_{1 / 2}\left(a_{e}\right) \\
& \zeta_{i}=d_{1 / 2}\left(a_{i}\right) \text { or } \quad g_{1 / 2}\left(a_{i}\right) .
\end{aligned}
$$

In the classical limit, $h \rightarrow 0,(7)$ and (9) reduce to Boltzmann statistics:

$$
\begin{aligned}
& n_{-+}(r)=n_{-0} \exp \left(-\beta w_{-+}(r)\right) \\
& n_{++}(r)=n_{+0} \exp \left(-\beta w_{++}(r)\right) .
\end{aligned}
$$


Classically, the average energies go to $w_{-+}(r) \rightarrow-z_{i} e^{2} / r$ and $w_{++}(r) \rightarrow+z_{i}^{2} e^{2} / r$ as $r$ approaches 0 . Consequently, $n_{++}(r)$ vanishes as $r$ approaches 0 , but $\mathrm{n}_{-+}(\mathrm{r})$ has an essential singularity. This catastrophic divergence renders the classical nonlinear Debye-Hückel equations inapplicable to a gas of point charges. The Fermi form (7) of $\mathrm{n}_{-+}(\mathrm{r})$ is well behaved at $\mathrm{r}=0$, since the repulsions due to the Pauli principle prevent the classical piling-up of negative charge around the ion.

By the same reasoning, the density of positive charge around an electron is given by:

$$
n_{+-}(r)=n_{+0} \zeta_{i}^{-1} \cdot \begin{cases}\ell_{1 / 2}\left(a_{i}-\beta w_{+-}\right) & \text {F.D. } \\ g_{1 / 2}\left(a_{i}-\beta w_{+-}\right) & \text {B.E. },\end{cases}
$$

and, similarly, for negative charge around the electron we have:

$$
n_{--}(r)=n_{-0} b_{e}^{-1} d_{1 / 2}\left(a_{e}-\beta w_{--}\right) \text {. }
$$

In order to obtain usable equations, it is next assumed that the various average energies are related to the average potentials as follows:

$$
\begin{aligned}
& w_{++}=z_{i} e \Psi_{+} \\
& w_{-+}=-e \Psi_{+} \\
& w_{+-}=z_{i} e \Psi_{-} \\
& w_{-}=-e \Psi_{-}
\end{aligned}
$$

The justification of this basic as sumption and the other approximations of the Debye-Hückel theory are discussed in detail by Fowler. 5

Kirkwood and Cowan form a nonlinear equation for $\Psi_{+}$by using (7) for $n_{-+}(r)$ and the Boltzmann form for $n_{++}(r)$. The equation for $\Psi_{-}$is coupled

${ }^{5}$ R. H. Fowler, Statistical Mechanics (Cambridge University Press, 1955), second edition, pp. $269-74$. 
to $\dot{\Psi}_{+}$by the use of the symmetry requirement (4). These equations are:

$$
\begin{array}{r}
\nabla^{2} \Psi_{+}=-4 \pi z_{i} \text { e } \delta(r)-4 \pi \text { el } z_{i} n_{+o} \zeta_{i}^{-1} \ell_{1 / 2}\left(a_{i}-\beta z_{i} \text { e } \Psi_{+}\right) \\
\left.-n_{-0} \zeta_{e}^{-1} l_{1 / 2}\left(a_{e}+\beta e \Psi_{+}\right)\right] \\
\nabla^{2} \Psi_{-}=4 \pi \text { e } \delta(r)-4 \pi \text { e[n-o } \zeta_{e}^{-1} \ell_{1 / 2}\left(a_{e}+\beta e \Psi_{+}\right) \\
\left.-n_{-o} \zeta_{e}^{-1} \cdot \ell_{1 / 2}\left(a_{e}+\beta e \Psi_{-}\right)\right] .
\end{array}
$$

(For generality, we have retained quantum statistics for the ions as well as electrons.) Using the same logic, however, one arrives at an alternative set of equations by first writing an equation for $\Psi_{-}$, and then coupling the equation for $\Psi_{+}$to that for $\Psi_{-}$with the symmetry requirement. This alternative set is:

$$
\begin{aligned}
& \nabla^{2} \Psi_{-}=4 \pi \text { e } \delta(r)-4 \pi \text { e }\left[z_{i} n_{+o} \zeta_{i}^{-1} d_{l / 2}\left(a_{i}-\beta z_{i} \text { e } \Psi_{-}\right)\right. \\
& \left.-n_{-0} \zeta_{e}^{-1} \cdot l_{1 / 2}\left(a_{e}+\beta e \Psi_{-}\right)\right] \\
& \nabla^{2} \Psi_{+}=-4 \pi z_{i} \text { e } \delta(r)-4 \pi \text { e }\left[z_{i} n_{+o} \zeta_{i}^{-1} \ell_{1 / 2}\left(a_{i}-\beta z_{i} \text { e } \Psi_{+}\right)\right. \\
& \left.-z_{i} n_{+o} \zeta_{i}^{-1} \ell_{1 / 2}\left(a_{i}-\beta z_{i} \text { e } \Psi_{-}\right)\right] \text {. }
\end{aligned}
$$

Both sets of equations, (15) and (16), reduce to the classical nonlinear equations of the Debye-Hückel theory in the classical limit, $\hbar \rightarrow 0$. For positively and negatively charged particles of comparable mass, there is no reason to prefer one set over the other. However, for real electrons and nuclei with their tremendous mass difference, the set (15) used by Kirkwood and Cowan seems more reasonable for approximate numerical computation of the thermodynamic functions of a dense plasma.

To examine the consequences of the two alternative sets of equations, we look at the solutions of their linearized forms. The linearized equations 
are valid for $\mathbf{r}^{\prime}$ sufficiently large so that $\beta z_{i}$ e $\Psi_{ \pm}$and $\beta$ e $\Psi_{ \pm}$are much greater than 1. The $d_{1 / 2}\left(\operatorname{or} g_{1 / 2}\right)$ functions may be expanded in Taylor series to give:

$$
\begin{aligned}
& \zeta_{e}^{-1} \ell_{1 / 2}\left(a_{e}+\beta e \Psi_{+}\right)=1+\beta e \theta_{e}^{2} \Psi_{+}+\cdots \\
& \zeta_{i}^{-1} d_{1 / 2}\left(a_{i}-\beta z_{i} e \Psi_{+}\right)=1-\beta z_{i} \text { e } \theta_{i}^{2} \Psi_{+}+\cdots
\end{aligned}
$$

where

$$
\begin{aligned}
& \theta_{e}^{2}=\zeta_{e}^{-1} d_{1 / 2}^{i}\left(a_{e}\right)=d_{-1 / 2}\left(a_{e}\right) / d_{1 / 2}\left(a_{e}\right) \\
& \theta_{i}^{2}=\zeta_{i}^{-1} \ell_{1 / 2}^{\prime}\left(a_{i}\right)=\ell_{-1 / 2}\left(a_{i}\right) / \ell_{1 / 2}\left(a_{i}\right)
\end{aligned}
$$

If (17) is used, the linearized equations obtained from (15) are:

$$
A\left\{\begin{array}{l}
\nabla^{2} \psi_{+}=4 \pi \beta \mathrm{e}^{2}\left[\mathrm{z}_{i}^{2} \theta_{i}^{2} \mathrm{n}_{+o}+\theta_{\mathrm{e}}^{2} \mathrm{n}_{-o}\right] \psi_{+} \\
\nabla^{2} \psi_{-}=4 \pi \beta \mathrm{e}^{2} \theta_{\mathrm{e}}^{2}\left[\mathrm{z}_{\mathrm{i}}^{2} \mathrm{n}_{+o} \psi_{+}-\mathrm{n}_{-o} \psi_{-}\right],
\end{array}\right.
$$

and from (16):

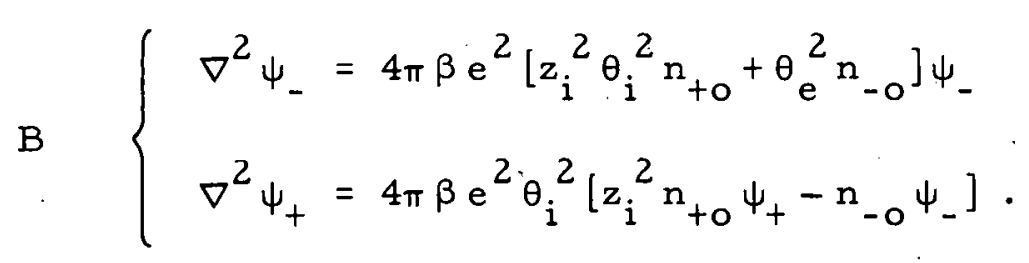

The solutions of the linearized equations are designated with $\psi_{+}$and $\psi_{-}$, to distinguish them from $\Psi_{+}$and $\Psi_{-}$for the nonlinear equations, where $\Psi_{+}$and $\Psi_{-}$are the asymptotic forms of $\Psi_{+}$and $\Psi_{-}$for large $r$. The $\delta$ functions expressing the boundary conditions at $r=0$ have not been witten in the linearized equations, since these boundary conditions are of no help in determining the integration constants in $\psi_{+}$and $\psi_{-}$.

The general solutions' of the linear equations $A$, subject only to the requirement that $\dot{\psi}_{+}$and $\psi_{-}$vanish at $\infty$, are: 


$$
\begin{aligned}
& \psi_{+}=\frac{z_{i} e}{r} C_{A+} e^{-k r} \\
& \psi_{-}=-\frac{e}{r}\left[C_{A-} e^{-k \theta_{-} e^{r}}+C_{A+}\left(\theta^{2} e^{2} \theta_{i}^{2}\right) e^{-k r}\right],
\end{aligned}
$$

where

$$
\begin{aligned}
k & =\left(k_{-}^{2} \theta_{e}^{2}+k_{+}^{2} \theta_{i}^{2}\right)^{1 / 2}=1 / \lambda \\
k_{-}^{2} & =4 \pi e^{2} \beta n_{-0} \\
k_{+}^{2} & =4 \pi e^{2} \beta z_{i}^{2} n_{+o} .
\end{aligned}
$$

Here, $\mathrm{C}_{\mathrm{At}}$ and $\mathrm{C}_{\mathrm{A}-}$ are arbitrary constants, and $k$ is the reciprocal of the Debye screening length $\dot{\lambda}$, generalized to include the modifications due to quantum statistics. Similarly, the gerieral solutions of equations $B$ are:

$$
\begin{aligned}
& \psi_{-}=-\frac{e}{r} C_{B-} e^{-k r} \\
& \psi_{+}=\frac{z_{i} e}{r}\left[C_{B+} e^{-k}+\theta_{i} r+C_{B-}\left(\theta_{i}^{2} / \theta_{e}^{2}\right) e^{-k r}\right] \text {. }
\end{aligned}
$$

By numerical integration of the nonlinear equations (15) and comparison of $\psi_{+}$and $\Psi_{-}$with $(21)$ for.large $r$, we may find numerical values for $\mathrm{C}_{\mathrm{A}+}$ and $C_{A-}$ - Similarly, numerical values of $C_{B+}$ and $C_{B-}$ could be found by numerical integration of (16). The solutions, (21) and (23), of the alternative sets of linear equations are different from each other, and hence would lead to different answers for the thermodynamic functions of the gas. Furthermore, $\psi_{+}$and $\psi_{\text {- }}$ have the unsatisfactory feature of behaving differently as $r$ approaches $\infty$. In (21), $\Psi_{+}$vanishes as $\mathrm{e}^{-k r}$, while $\psi_{-}$vanishes as $e^{-\kappa^{6} \theta^{r}} e^{r}$, since $\kappa_{-} \theta_{e}$ is less than $\dddot{k}$.

The only way in which the solutions (21) and (23) can be identical is to have the condition: $C_{A_{-}}=C_{B+}=0$. If this requirement is made, then (20) and (23) have the same form and require only one arbitrary constant: 


$$
\begin{aligned}
& \psi_{+}=\frac{z_{i} e \theta_{i}^{2}}{r} C e^{-k r} \\
& \psi_{-}=-\frac{e \theta_{e}^{2}}{r} C e^{-k r} .
\end{aligned}
$$

In view of the nonuniqueness of the theory, as illustrated by two apparently equally valid sets of equations $A$ and $B$, the solutions (24) are to be preferred over (21) or (23). In the classical limit, the degeneracy parameters $\theta_{i}^{2}$ and $\theta_{e}^{2}$ both reduce to 1 . The remaining arbitrary constant $C$ is determined by the fact that the Debye-Hückel theory is rigorously valid at sufficiently high temperature. The asymptotic forms $\psi_{+}$and $\psi_{-}$are valid arbitrarily close to the origin, and at the origin we must have $r \Psi_{+}$equal to $z_{i}$ e and $r \Psi_{\text {_ }}$ equal to - e; hence, $C$ has the value 1 for a nondegenerate gas. It is clear, however, that for a degenerate gas (i.e., $\theta_{e}^{2} \neq \theta_{i}^{2} \neq 1$ ) the constant $C$ cannot be chosen so that $\psi_{+}$and $\psi_{-}$reduce to $z_{i} e / r$ and $-e / r$, respectively, as $r$ approaches 0 .

The chief criticism of the two sets of nonlinear equations, (15) and (16), for the calculation of thermodynamic functions of a dense gas is that they are unsymmetrical in the way $\Psi_{+}$and $\Psi_{-}$are treated. With the set of equations (14) used by Kirkwood and Cowan, $\Psi_{+}$may be calculated independently of $\Psi_{-}$, but the calculation of $\Psi_{-}$requires a knowledge of $\Psi_{+}$. The same statement applies to the equations.(16), but in reverse order. The two ways of doing the calculation, in general, will give different results. Thus, it would seem more reasonable to begin with a symmetrical set of equations in which $\Psi_{+}$and $\Psi_{-}$ are treated on an equal basis. The first equation of (15) and the first of (16) are symmetrical in this sense. An objection against this symmetrical set of equations for numerical calculations of the type done by Kirkwood and Cowan is that the symmetry requirement, $n_{-+}(r)=z_{i} n_{+-}(r)$, is violated for $r$ small enough to cause nonlinear effects to be important. We will show, however, 
that the symmetrical equations are suitable for developing a linearized Debye-. Hückel theory which gives the correct results for the thermodynamic functions

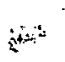
of a slightly degenerate gas.

The linearized symmetrical equations, namely, the first of $A$ and the first of $B$, have the solutions:

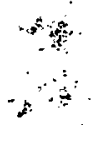

$$
\begin{aligned}
& \psi_{+}=\frac{z_{i} e}{r} C_{+} e^{-k r} \\
& \psi_{-}=-\frac{e}{r} C_{-} e^{-k r} .
\end{aligned}
$$

The symmetry requirement, $n_{-+}(r)=z_{i} n_{+-}(r)$, gives a relation between the two arbitrary constants $\mathrm{C}_{+}$and $\mathrm{C}_{-}$in (25). The expansions of these charge densities are:

$$
\begin{gathered}
n_{-+}(r)^{\prime}=n_{-o}\left\{1+\beta e \theta_{e}^{2} \psi_{+}+\ldots\right. \\
z_{i} n_{+-}(r)=z_{i} n_{+o}\left\{1-\beta e \theta_{i}^{2} \psi_{-}+\ldots .\right.
\end{gathered}
$$

The two densities, $\mathrm{n}_{-+}$and $\mathrm{zn}_{+_{-}}$, can be equal to $0(\psi)$ only if $\theta_{\mathrm{e}}{ }^{2} \mathrm{C}_{+}$is equal to $\theta_{i}^{2} C_{-}$, and (25) may then be written as (24). Thus, the equality of the solution of the unsymmetrical sets of linearized equations, $A$ and $B$, is equivalent to preserving the charge-density symmetry in $0(\psi)$. The symmetry is violated in $O\left(\psi^{2}\right)$ for a degenerate gas, and hence the usefulness of the nonlinear symmetrical equations for numerical calculations is questionable.

Landau and Lifshitz, in their discussion of the degenerate plasma, used the symmetrical linearized equations and obtained the solutions (25). They used the boundary conditions at $r=0$, however, and so put the constants $\mathrm{C}_{+}$ and $C_{\text {_ }}$ equal to 1 . This choice violates the symmetry requirement, even to first order in $\psi$. 


\section{CALCULATION OF THE THERMODYNAMIC FUNCTIONS}

The average potential energy of the gas is given by:

$$
\begin{aligned}
\bar{U} & =\frac{1}{2} N_{i} \int d \underset{\sim}{r} \rho_{+}\left(z_{i} e / r\right)+\frac{1}{2} N_{e} \int d \vec{r} \rho_{-}(-e / r) \\
& =\bar{U}_{i}+\bar{U}_{e} .
\end{aligned}
$$

The solutions of the linearized equations (24) are valid for sufficiently large $r$, and with Poisson's equations, they give the asymptotic forms of the charge densities as:

$$
\begin{aligned}
& \rho_{+ \text {asy }}=-\frac{1}{4 \pi} \nabla^{2} \psi_{+}=-\frac{z_{i} e \theta_{i}^{2} 2}{r} C^{-k x r} \\
& \rho_{\text {- asy }}=-\frac{1}{4 \pi} \nabla^{2} \psi_{-}=\frac{e \theta_{e}^{2} 2}{r} C e^{-k r} .
\end{aligned}
$$

The asymptotic forms (28) are accurate for distances from the central point charges greater than some limit to be denoted as $r_{c}$. Inside the sphere of radius $r_{c}$, the average potential and charge density are not known, though presumably they may be calculated approximately from the nonlinear equations, either (15) or (16). The average energy integral is thus a sum of two parts:

$$
\bar{U}_{i}=\frac{1}{\lambda} N_{i} 4 \pi \int_{0}^{r} c d r r^{2} \rho_{+ \text {exart }}\left(z_{i} e / r\right)+\frac{1}{2} N_{i} 4 \pi \int_{r_{c}}^{\infty} d r r^{2} \rho_{+a 0 y}\left(z_{i} e / r\right) .
$$

The quantity $\bar{U}_{i}$ may be estimated by using $\rho_{+}$asy for the entire volume, even for $r$ less than $r_{c}$ where it is not accurate, since the main contribution comes from $r$ greater than $r_{c} \cdot$ With this approximation and using (28), we find:

$$
\bar{U}_{i} \cong \frac{1}{2} N_{i} 4 \pi \int_{0}^{\infty} d r r^{2} \rho+a s y\left(z_{i} e / r\right)=-\frac{1}{2} C N_{i} z_{i}^{2} e^{2} \theta_{i}^{2} k \text {. }
$$

Adding a similar result for $\bar{U}_{e}$, we have, for the potential energy of the gas due to the particle correlation, 


$$
\begin{aligned}
\bar{U} / V & =-\frac{1}{2} C e^{2}\left(z_{i}^{2} \theta_{i}^{2} n_{+o}+\theta_{e}^{2} n_{-o}\right) k \\
& =-\frac{C k T k^{3}}{8 \pi} .
\end{aligned}
$$

We have seen that the remaining arbitrary constant $C$ must be 1 in the classical limit, but for a degenerate gas, we cannot rule out the possibility that $C$ is a function of temperature in such a way that $\lim C(T)$ is equal to 1 as $T$ approaches $\infty$. Equation (31), however, agrees with the exact near-classicallimit result for $\bar{U}$ as derived from the grand partition function (Eq. 122 of the previous paper) when $C$ is equal to 1.

The Helmholtz free energy may be obtained from $\bar{U}$ by an integration with respect to the coupling constant from 0 to $e^{2}$; this calculation is equivalent to the Debye, charging process. Noting that $\bar{U}$ is $0\left(e^{3}\right)$, we easily find the contribution to the Helmholtz free energy to be:

$$
F_{e}=\int_{0}^{e^{2}} \frac{d\left(e^{1^{2}}\right)}{\left(e^{12}\right)} \bar{U}_{e^{\prime}}=-\frac{V k T k^{3}}{12 \pi},
$$

or, in the notation of the previous paper,

$$
\frac{F_{\mathrm{e}}}{\mathrm{kT}}=-\frac{2}{3} \mathrm{~N} \Lambda \phi^{3 / 2}
$$

where

$$
\begin{aligned}
& N=N_{e}+N_{i}, \quad n=n_{+o}+n_{-o}, \quad f_{e}=N_{e} / N \\
& \Lambda=\sqrt{\pi} e^{3} \beta^{3 / 2}{ }_{n}^{1 / 2}, \quad \phi=z_{i}^{2} \theta_{i}^{2} f_{i}+\theta_{e}^{2} f_{e} .
\end{aligned}
$$

If the free energy due to the interaction is added to $F_{0}$, the free energy of an ideal quantum gas which is given by:

$$
\frac{F_{0}}{\mathrm{kT}}=\mathrm{N}\left\{a-\ell_{3 / 2}(a) / \ell_{1 / 2}(a)\right\}
$$


then the total free energy is given by:

$$
F=F_{o i}+F_{o e}+F_{e} \text {. }
$$

The pressure and internal energy are obtained from the expressions:

$$
\begin{aligned}
& \frac{P V}{k T}=-V \frac{\partial}{\partial V}\left(\frac{F}{k T}\right) \\
& \frac{E}{k T}=\beta \cdot \frac{\partial}{\partial \beta}\left(\frac{F}{k T}\right) .
\end{aligned}
$$

The differentiations of (36) are easily carried out by noting that from the defining relation for the chemical potential (10) we obtain:

$$
\begin{aligned}
& \mathrm{V} \partial \mathrm{a} / \partial \mathrm{V}=-1 / \theta^{2} \\
& \beta \partial a / \partial \beta=3 / 2 \theta^{2} .
\end{aligned}
$$

Also, we need the relation:

$$
\begin{gathered}
v \frac{\partial}{\partial V}\left(\frac{\ell^{-1 / 2^{(a)}}}{\ell_{1 / 2^{(a)}}}\right)=\left[\frac{\ell_{-3 / 2^{(a)}}}{\ell_{1 / 2^{(a)}}}-\frac{\ell_{-1 / 2^{(a)}}^{2}}{d_{1 / 2^{2}(a)}^{2}}\right] v \frac{\partial a}{\partial v} \\
\quad=-\left(\frac{\ell_{-1 / 2^{(a)}}}{\ell_{1 / 2^{(a)}}}-\frac{\ell_{-3 / 2^{(a)}}}{\ell_{-1 / 2^{(a)}}}\right) \doteq-\eta(a) .
\end{gathered}
$$

From (36), using (37) and (38), we obtain the following expressions for the pressure and energy:

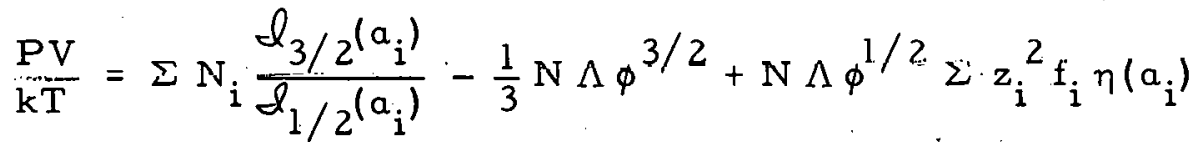

$$
\begin{aligned}
& \frac{E}{k T}=\frac{3}{2} \Sigma N_{i} \frac{\ell_{3 / 2}\left(a_{i}\right)}{\ell_{1 / 2}\left(a_{i}\right)}-N \Lambda \phi^{3 / 2}+\frac{3}{2} N \Lambda \phi^{1 / 2} \Sigma z_{i}{ }^{2} f_{i} \eta\left(a_{i}\right),
\end{aligned}
$$

where the sums are over the particle species of a multicomponent gas. Expressions (39) are identical with (127) and (128) of the previous article, except for the absence of the exchange interaction term. 


\section{CONCLUSION}

The pressure and internal energy formulae (39) are exact in the nearclassical limit defined by high temperature and low density such that $\Lambda_{\phi}^{3 / 2}=$ $\left(8 \pi \rho \lambda^{3}\right)^{-1}<1$, but $\hbar \neq 0$. In this limit, the ring diagrams of the expansion of the quantum-mechanical grand partition function give the leading contribution to $\mathrm{PV} / \mathrm{kT}$ after the ideal gas term (namely, $O(\Lambda)$ ), and the next contribution from non-ring terms is $O\left(\Lambda^{2}\right)$. We have thus shown that the linearized DebyeHückel-Thomas-Fermi theory is exact in the near-classical limit in the same way in which the Debye-Hückel theory is exact in the classical limit $(\hbar=0)$. To distinguish between the various ways of building up such a theory, we must require that the Poisson equations be symmetrical in the treatment of the average potentials, and preserve the charge-density symmetry, $n_{-+}=z_{i} n_{+-}$to $0(\psi)$. The usefulness of the Debye-Hückel method has thus been extended to more dense plasmas, but at the same time the limitations of the method are more apparent. The linearized theory gives the thermodynamic functions of essentially classical particles whose velocity distributions are slightly modified from the Boltzmann form, due to quantum statistics. Exchange interactions and wave-mechanical effects are in no way included in either the linear or the nonlinear equations. Furthermore, it is not clear that the nonlinear equations give any more information than is contained in the linearized forms. The nonlinear effects of the symmetrical nonlinear equations (the final equation of (15) and the first of (16) are not believable, since the charge symmetry, $n_{-+}=z_{i} n_{+_{-}}$, is violated in $0\left(\psi^{2}\right)$. The set of nonlinear equations (15) used by Cowan and Kirkwood are better in that the clidrge symmetry is preserved, but the set $(16)$, which gives different answers, would seem to be equally valid. Until more exact results have been obtained from the non-ring terms of the partition function, and can be used for comparison, we conclude that the' Debye-Hückel-Thomas-Fermi theory, as applied to plasmas, is useful only in its linearized form. 